\title{
Multi-dimensional modelling of spray, in-cylinder air motion and fuel-air mixing in a direct-injection engine
}

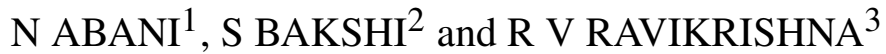 \\ Department of Mechanical Engineering, Indian Institute of Science, \\ Bangalore 560012 \\ *e-mail: ravikris@mecheng.iisc.ernet.in
}

Ms received 29 September 2006; revised 10 May 2007

\begin{abstract}
In this work, three-dimensional fuel-air mixing inside a conventional spark ignition engine cylinder is simulated under direct injection conditions. The motivation is to explore retrofitting of conventional engines for direct injection to take advantage of low emissions and high thermal efficiency of the direct injection concept. Fuel-air mixing is studied at different loads by developing and applying a model based on the Lagrangian-drop and Eulerian-fluid (LDEF) procedure for modelling the two-phase flow. The Taylor Analogy Breakup (TAB) model for modelling the hollow cone spray and appropriate models for droplet impingement, drag and evaporation are used. Moving boundary algorithm and two-way interaction between both phases are implemented. Fuel injection timing and quantity is varied with load. Results show that near-stoichiometric fuel-air ratio region is observed at different locations depending on the load. The model developed serves to predict the fuel-air mixing spatially and temporally, and hence is a useful tool in design and optimization of direct injection engines with regards to injector and spark plug locations. Simulations over a range of speed and load indicate the need for a novel ignition strategy involving dual spark plugs and also provide guidelines in deciding spark plug locations.
\end{abstract}

Keywords. SI engine; direct injection; in-cylinder fuel-air mixing; CFD; two-phase flow.

\section{Introduction}

Direct Injection (DI) of petrol/gasoline has been an emerging and successful technology in lowering emissions and improving the thermal efficiency of engines. Jost (2001) mentions about fuel-stratified injection (FSI) used in a recent engine design of Volkswagen, wherein

\footnotetext{
${ }^{1}$ Current address: University of Wisconsin-Madison, USA

${ }^{2}$ Current address: Department of Mechanical Engineering, Indian Institute of Technology, Chennai

${ }^{3}$ Corresponding author
} 
fuel savings of up to $15 \%$ and reduction in emissions have been achieved. There is great interest in the potential for retrofitting existing engines for direct fuel injection to take advantage of the higher efficiency and lower emissions. It has been widely known that the engine performance and emissions are dependent on the initial fuel vapour distribution in the cylinder. Hence, the location of the injector, air motion and fuel delivery characteristics become important factors for study.

The efficiency of a DI engine depends on the mixture preparation and its distribution inside the chamber. This phenomenon is based on the interaction of the in-cylinder air motion, either tumble or swirl, and the spray characteristics from the high pressure swirl injector which are generally employed for these engines. The location of injector, spark plug, piston geometry, cylinder geometry and injection timing are important criteria in the design of DI engines. These engines have potential to achieve greater fuel economy compared with a diesel engine at partial loads and give better performance than port fuel injection (PFI) spark ignition engines at high loads. The thermodynamic potential for reducing specific fuel consumption (sfc), coupled with the advantages of quicker starting, enhanced transient response, and more precise control of the mixture air-fuel ratio, have resulted in a renewed worldwide interest in DI engines as an automotive power plant.

The simplest DI engine system is the one that operates in the early-injection, homogeneous, stoichiometric mode. This engine must utilize throttling for load control, thus the full fuel economy associated with the elimination of throttling will not be realized. Engines operating in this mode however, do experience the thermodynamic advantages of intake charge cooling and rapid starting and fuel cut-off on deceleration. Next level of complexity involves using a leaner homogeneous mixture with reduced throttling for some degree of load control. Making use of the full potential of the DI concept requires a design that generates a stable, stratified mixture using an overall lean air-fuel ratio for operating at part load, and which can undergo a smooth transition to heavy or full load operation by injecting increased volumes of fuel at earlier times in the cycle.

In this paper, the objectives are to predict the spatial and temporal distribution of fuel-air mixing inside the cylinder under conditions of direct injection of petrol/gasoline. A threedimensional model for simulating the two-phase flow with two-way coupling is developed and applied. Two-way coupling implies that the effect of the surrounding air motion on the droplets, and the effect of the droplets on the air motion are accounted for. Simulations are performed for different load-speed conditions. Based on results of the simulation, a novel ignition methodology involving use of dual spark plugs is suggested. The following sections present details of the engine configuration, the methodology adopted, operating conditions, and results of the simulations.

\section{Engine configuration}

The engine chosen is a conventional flat-piston design into which gasoline is injected directly through an injector that gives a hollow cone fuel spray. The timing and amount of fuel injection depends on the load at the engine. At low loads, the fuel is injected late, i.e., in the latter part of the compression stroke so as to create a stratified mixture to save fuel and ensure that the engine continues to run with the benefit that the mixture is near-stoichiometric near the spark plug location as suggested by Iwamoto et al (1997). At high loads, the fuel is injected early in the suction stroke to allow the fuel droplets to mix with the in-cylinder air and create a homogeneous mixture at the end of compression. DI engines usually employ a hollow cone fuel injector. This gives a wider spread of the spray and droplet dispersion. 


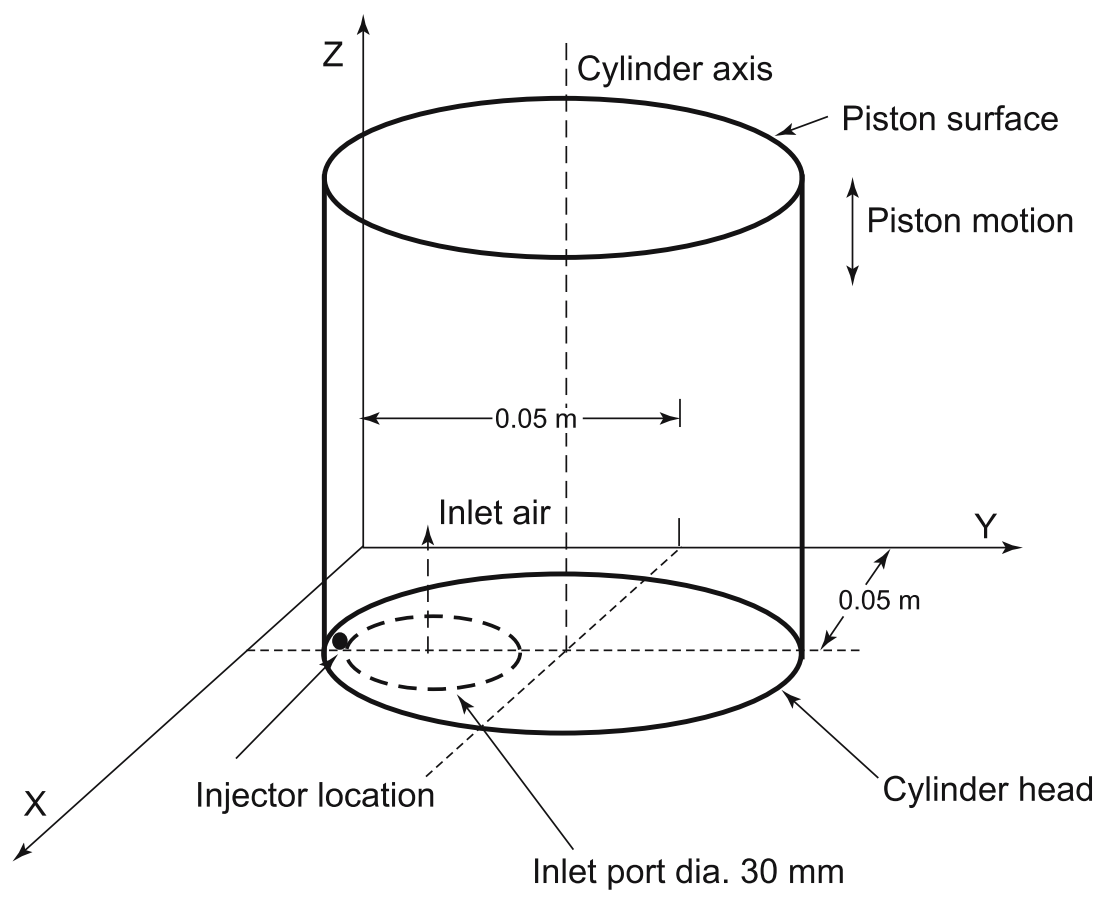

Figure 1. Schematic of the computational domain.

Since the objective is to explore the retrofitting of conventional engines, a flat piston geometry as shown in figure 1 was chosen. The bore of the cylinder is $80 \mathrm{~mm}$ and the stroke is $88 \mathrm{~mm}$. The intake port was a spherical hole of diameter $20 \mathrm{~mm}$ at the flat top cylinder head towards the sidewall and away from the cylinder center. In the present case, the location of fuel injector is fixed and is located near the inlet port with a slight offset distance. The fuel injector is oriented at an angle of $45^{\circ}$ with respect to the cylinder axis and is in a plane passing through both the injector and the axis of the cylinder. The layout of the cylinder and the computational domain are also shown in figure 1 . The solid wall of the cylinder is simulated by specifying a very high value of viscosity, since it is included in the computation domain. The full computation domain was $100 \mathrm{~mm} \times 100 \mathrm{~mm}$ in $\mathrm{x}-\mathrm{y}$ plane and $20 \mathrm{~mm}$ at $18^{\circ}$ of crank angle in z-direction. The in-cylinder domain in the $x-y$ plane has a dimension of $80 \mathrm{~mm}$. Total grid size was $30 \times 40 \times 40$ with 30 cells in z-direction and 40 each in $\mathrm{x}$-direction and $\mathrm{y}$ direction. The compression ratio of the engine was 10.5:1. The computation was performed over $324^{\circ}$ of crank angle, starting from $18^{\circ}$ ATDC to $18^{\circ}$ BTDC. Other details regarding the operating conditions are listed in table 1.

\section{Model description and numerical methodology}

The main objective here is to study the two-phase flow and the distribution of the fuel-vapour air mixture. A comprehensive three-dimensional model is developed using the Lagrangiandrop and Eulerian-fluid (LDEF) approach. The spray droplets are modelled in a statistical approach where discrete droplets are tracked using a Lagrangian approach. The gas phase is modelled in the Eulerian approach, which is the conventional continuum approach using 
Table 1. Engine details.

Engine and computational details

Compression ratio

Intake pressure (MPa)

$10 \cdot 5$

$0 \cdot 11$

Intake gas temperature $(\mathrm{K})$

320

Temperature inside cylinder at start (K)

550

380

380

Cylinder head temperature (K)

380

Cylinder liner temperature $(\mathrm{K})$

Fuel

Start of computation

End of computation

Start of suction

End of suction

Inlet velocity of air during suction $(\mathrm{m} / \mathrm{s})$

Iso-octane

$18^{\circ}$ ATDC

$18^{\circ} \mathrm{BTDC}$

$18^{\circ}$ ATDC

$9^{\circ} \mathrm{ABDC}$

120

Distance between inlet port center and cylinder liner wall (mm) 19

Distance between fuel injector and the cylinder top head ( $\mathrm{mm})$

19

Diameter of inlet port

$30 \mathrm{~mm}$

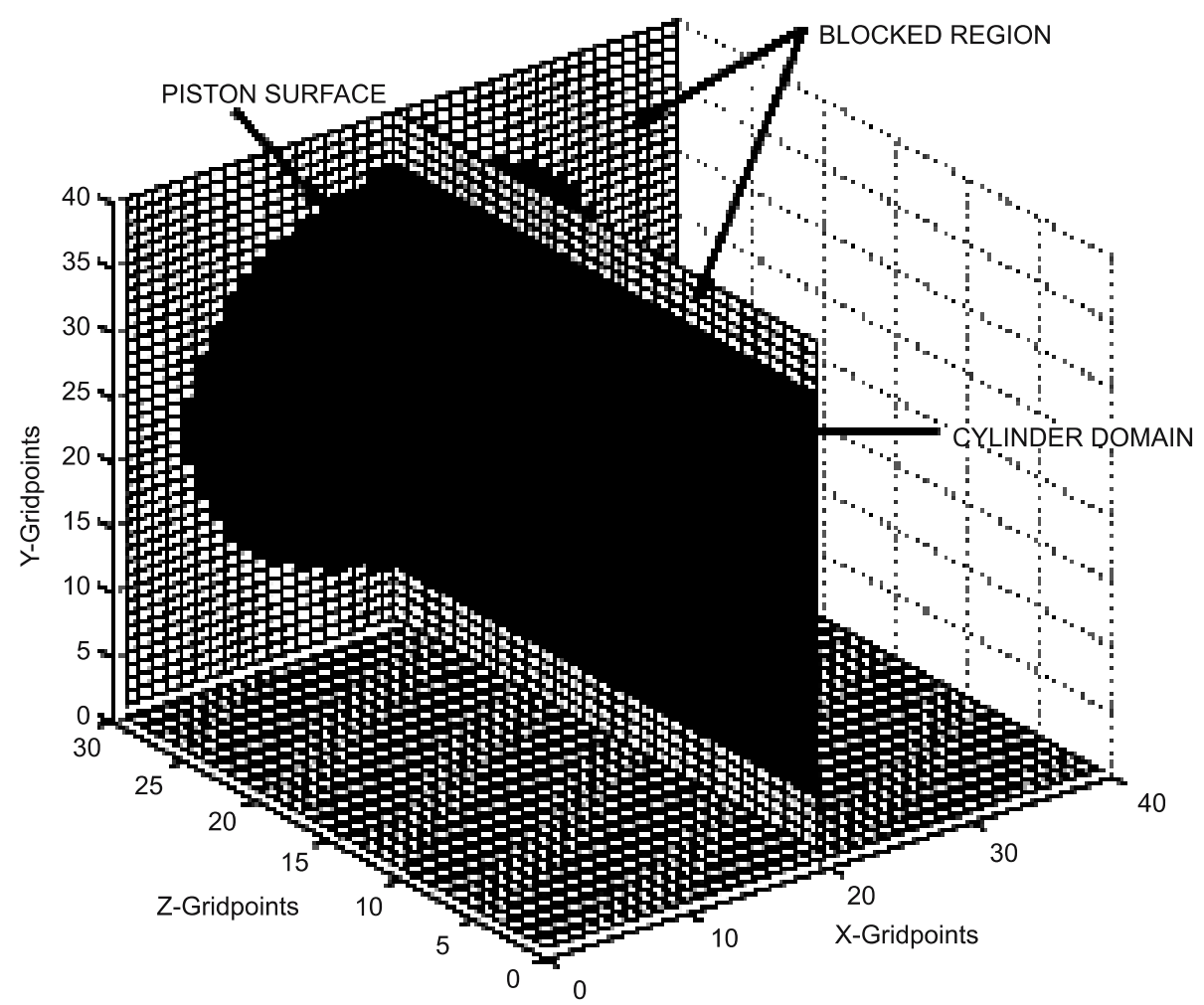

Figure 2. Mesh generated for the three-dimensional engine simulation. 


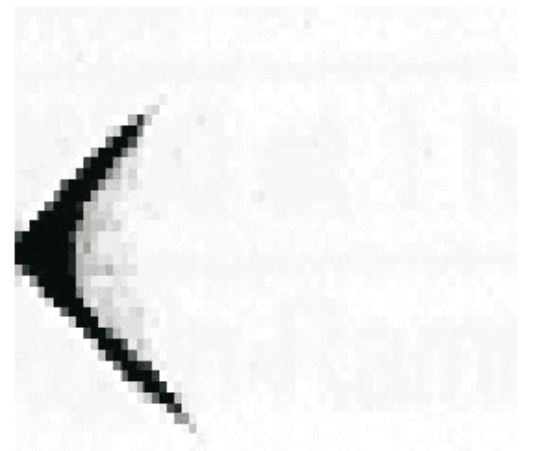

(a)

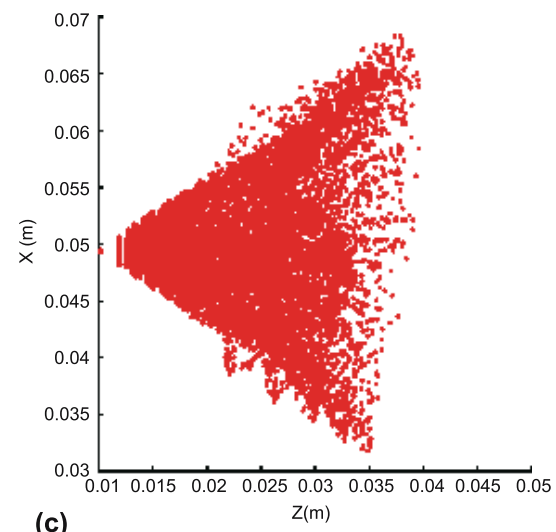

(c)

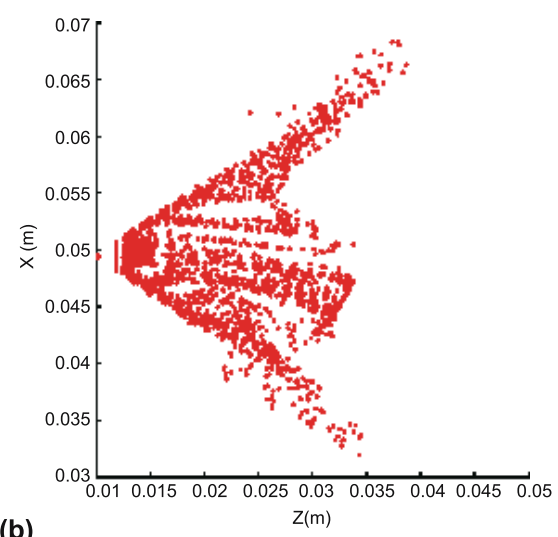

(b)

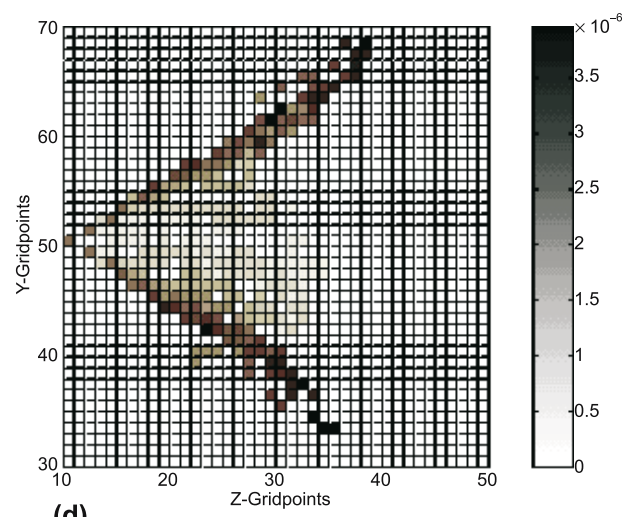

(d)

Figure 3. At $t=0.6 \mathrm{~ms}$ : (a) Laser sheet image showing spray structure; (b) Predicted spray structure along the same plane as that of the experimental image; (c) Predicted overall spray structure showing locations of all droplet parcels; (d) SMD distribution in the same plane as that of the experimental image. The grayscale bar on the right indicates the SMD in $\mathrm{m}$.

Finite Volume Method (FVM). The interaction of the two phases is achieved by coupling the respective mass, momentum and energy conservation equations. The effect of the gas phase on liquid phase is achieved by considering relative terms and gradients for mass, momentum and energy. The transfer of thermal energy is towards the droplet from the surrounding gas phase. With this gain in thermal energy, the droplet temperature increases. At the droplet temperature, the fuel-vapour concentration at the droplet surface is considered saturated. The difference in the concentration at the droplet surface and in the surrounding gas phase causes a concentration gradient. This results in mass transfer from the droplet surface to the surrounding gas phase. Similarly, the transfer of momentum to either of the phase is a two-way phenomenon and the momentum lost by the droplets is gained by the surrounding gas phase and similarly the momentum of the gas phase causes change in momentum of the droplets. The governing equations of flow, the spray model equations and the moving boundary algorithm are not detailed here for want of space; however, a short description is given below. Details of the gas-phase model incorporating the moving boundary algorithm can be found in an earlier work (Bakshi \& Ravikrishna 2003). 

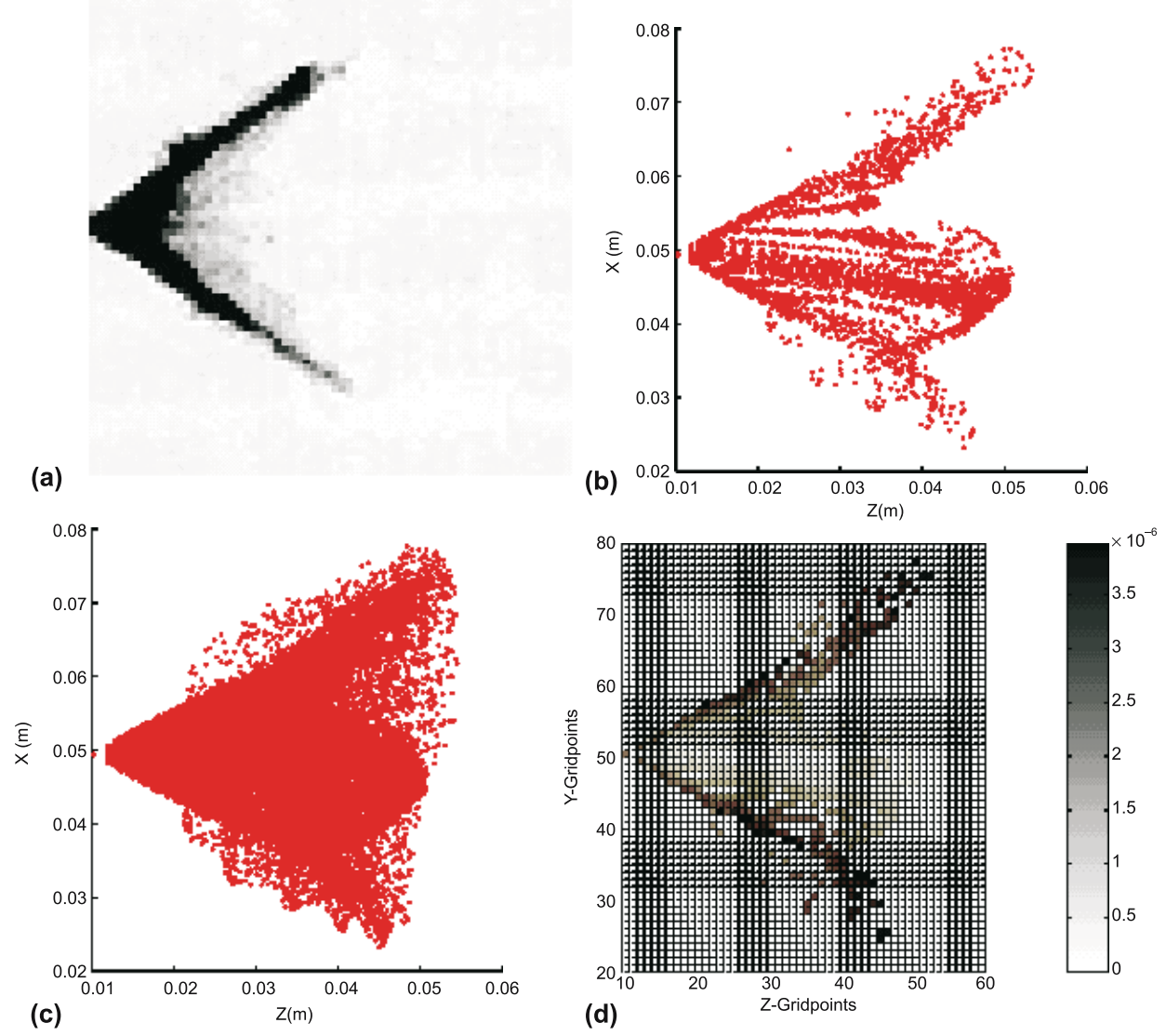

Figure 4. At $t=1.2 \mathrm{~ms}$ : (a) Laser sheet image showing spray structure; (b) Predicted spray structure along the same plane as that of the experimental image; (c) Predicted overall spray structure showing locations of all droplet parcels; (d) SMD distribution in the same plane as that of the experimental image. The grayscale bar on the right indicates the SMD in $\mathrm{m}$.

\subsection{Spray model}

The spray model includes tracking of droplets in a Lagrangian manner, which constitute the hollow cone fuel spray. The approach adopted here is a discrete blob approach where set of blobs emerges from the injector as proposed by Han et al (1997). These blobs are placed along the circumference of the injector and are given an initial velocity and flow direction depending on the sheet velocity and swirl angles. The swirling flow is provided by providing direction cosines based on the tangential and radial angle of the spray.

These blobs are subjected to break-up based on the assumption that the blobs oscillate and distort about their equator and break into smaller sizes once the break-up criteria is satisfied. The break-up model implemented was adopted from the works of O'Rourke \& Amsden (1987), which is based on Taylor's Analogy Break-up (TAB) mechanism (1963). Collision and Coalescence is neglected, as its probability in an evaporating environment for a hollow cone fuel spray is minimal since the droplets soon spread over the large area. The blobs are not subjected to drag, as the chunk of liquid mass, which is represented by the blobs, will have 


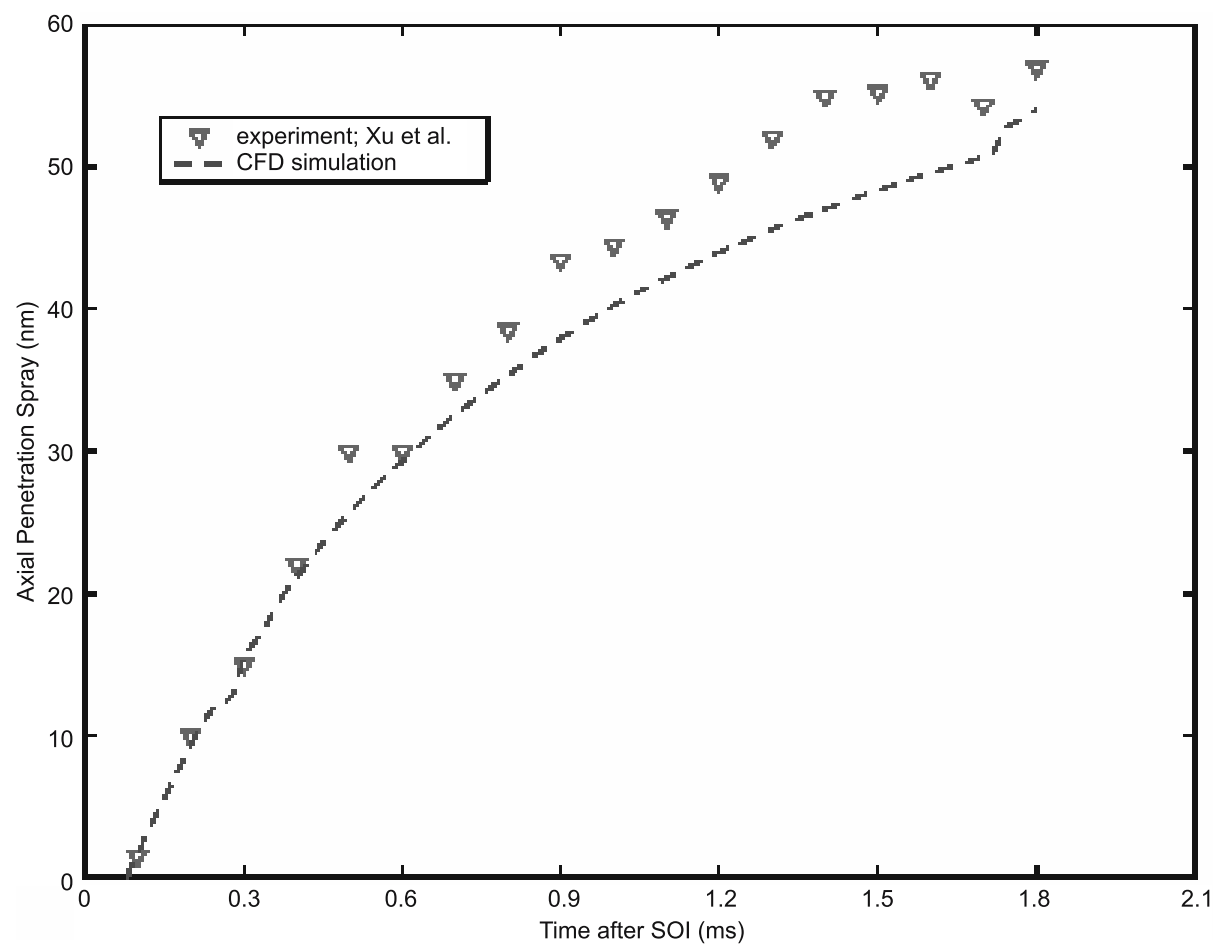

Figure 5. Spray tip penetration variation with time.

negligible drag and evaporation. However, once the blobs break-up into smaller droplets, the droplets are subjected to drag and evaporation.

An impingement model accounting for droplet impingement model on a flat wall has been considered that depends on the wall temperature. The spray impingement is modelled for a high temperature dry wall as found in the works of Senda et al (1994). This considers heat transfer from the wall to the droplet, the breakup behaviour of impinging droplets. To account for the dispersion process of newly formed droplets, impingement model of Naber and Reitz (1988) has been used.

\subsection{Gas phase model}

The mesh generated in the domain of the gas phase simulation is shown in figure 2. The gas phase here is the in-cylinder air motion is modelled using an Eulerian approach and is solved for fuel vapour-air mixture. To model the in-cylinder air motion SIMPLE algorithm (Patankar 1980) in conjunction with modification suggested by Doormal and Raithby (1984) is used to solve the coupled conservation equation for three-dimensional compressible flow. The piston motion is modelled using a moving boundary approach (Ferziger \& Peric 2003), and a pseudo flow velocity (Heywood 1988) approach is used to model the flow through the intake valve. Inclusion of moving boundary approach modified the standard Navier-Stokes equation by the inclusion of the convective flux taking into account using the relative velocity of the fluid with respect to the piston motion. The gas phase gains mass as the droplet evaporates. The lost fuel-vapour is added to the corresponding gas phase control volume as a source term in the 
continuity equation and the species equation. Similarly, the momentum lost by the droplet is fed to the corresponding control volume as the rate of change of the droplet momentum in that control volume. Since combustion is not modelled here, energy coupling is not implemented, as its effect will be very minimal.

\subsection{Model validation}

This section describes the validation of the two-phase model with experiments from literature (Xu \& Markle 1998). For the hollow cone spray, an outwardly opening injector with no sac volume is selected. The mass flow rate through this type of injectors is fairly constant throughout the injection pulse. These injectors give very fine drop sizes and hence, shorter penetration. A square waveform is assumed for the injection, i.e., a constant mass flow rate. A mass of $14 \mathrm{mg}$ of gasoline was injected for $1.3 \mathrm{~ms}$ at a rail pressure of $10 \mathrm{MPa}$. This section describes the validation of our model predictions for the spray by comparing with experimental laser sheet images of the spray from the work of Xu \& Markle (1998). The scanned laser sheet images are compared with model predictions along a single plane. These comparisons are shown in figures 3 and 4. Each figure has four parts, a,b,c and d. Part (a) is a laser sheet image of the spray structure in a plane passing through the injector, from the experiments of Xu and Markle (1998). Part (b) is the spray structure from the simulation performed in a plane passing through the axis of the spray and the injector. Part (c) represents the overall spray structure showing locations of all parcels and part (d) shows the predicted variation of SMD of the spray in a plane passing through the axis of the spray. This plane is the same as the one corresponding to the experimental data in Part (a).

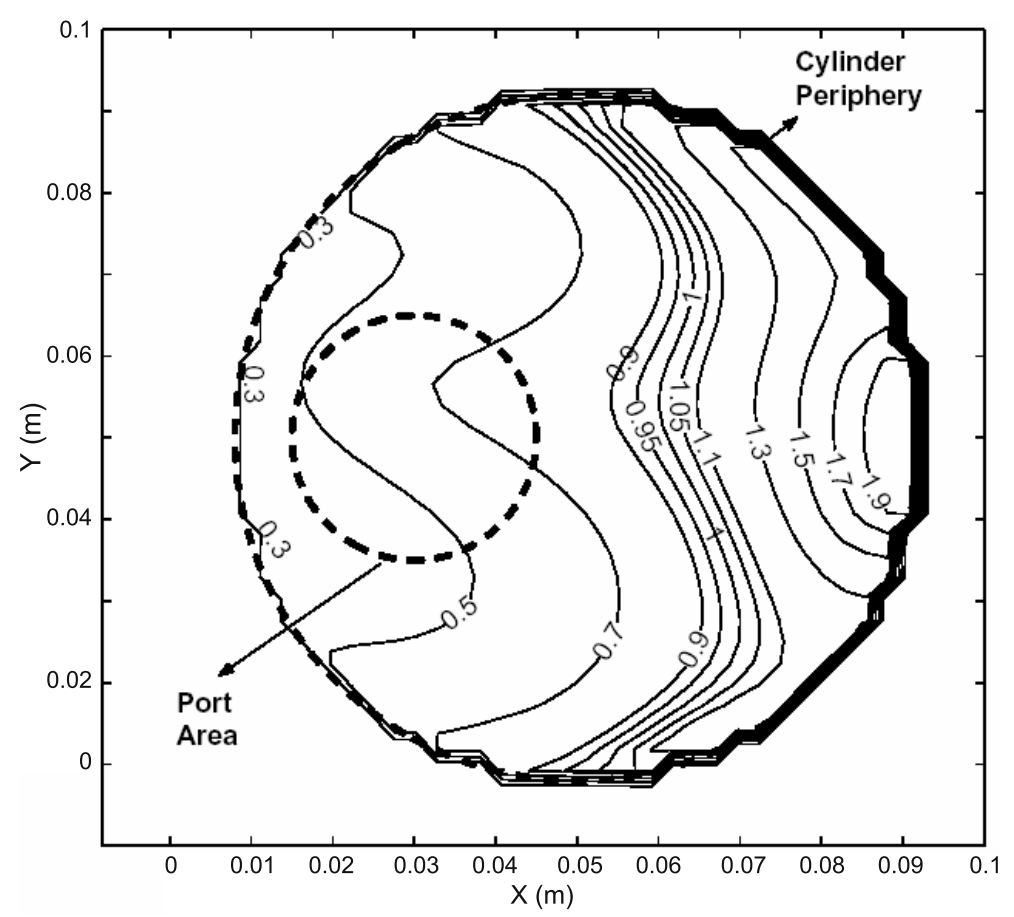

Figure 6. Case 1: Distribution of equivalence ratio in a plane near cylinder head at $18^{\circ}$ BTDC. 


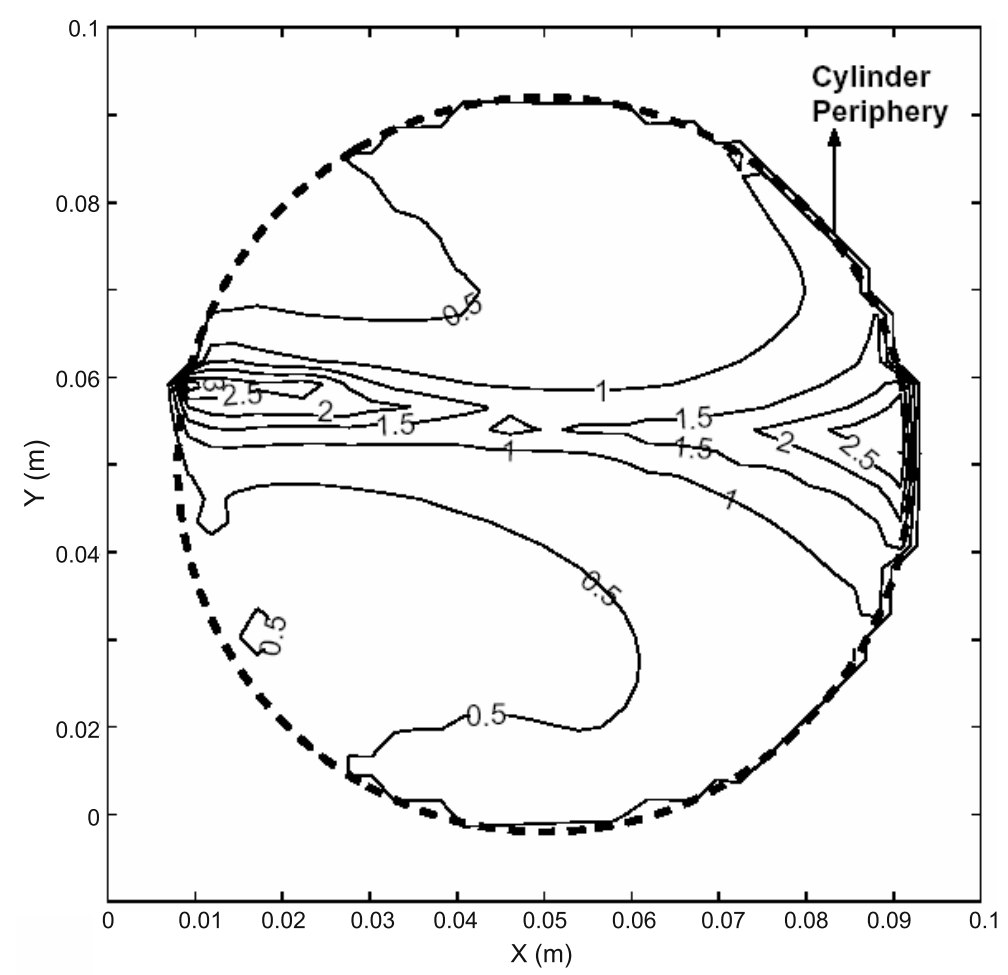

Figure 7. Case 1: Distribution of equivalence ratio in a plane near the piston surface at $18^{\circ} \mathrm{BTDC}$.

Figure 3 is at $0.60 \mathrm{~ms}$ after the start of injection (SOI). The smaller droplets are observed to move towards center of the spray due to the gas velocity induced by the spray. The larger droplets continue to travel in the original direction. This is clear from the SMD information in part (d) of the same figure. Experimental results in part (a) show similar trends. Since the laser light intensity is proportional to the droplet size, the very low signal in the central core region indicates very small or no droplets. This is in agreement with predictions as observed from the SMD information in part (d) of the same figure. This is evident from the spray structure

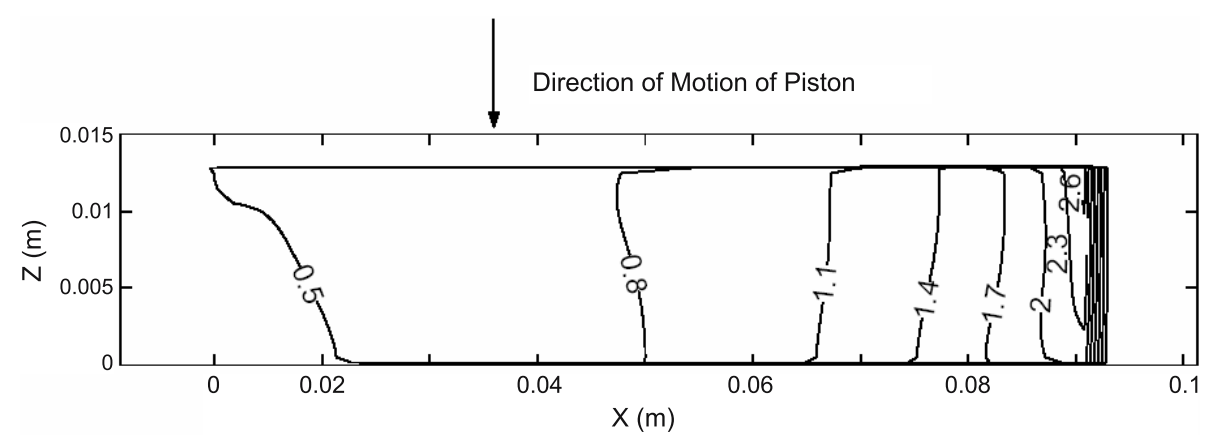

Figure 8. Case 1: Distribution of equivalence ratio in the central plane at $18^{\circ} \mathrm{BTDC}$. 


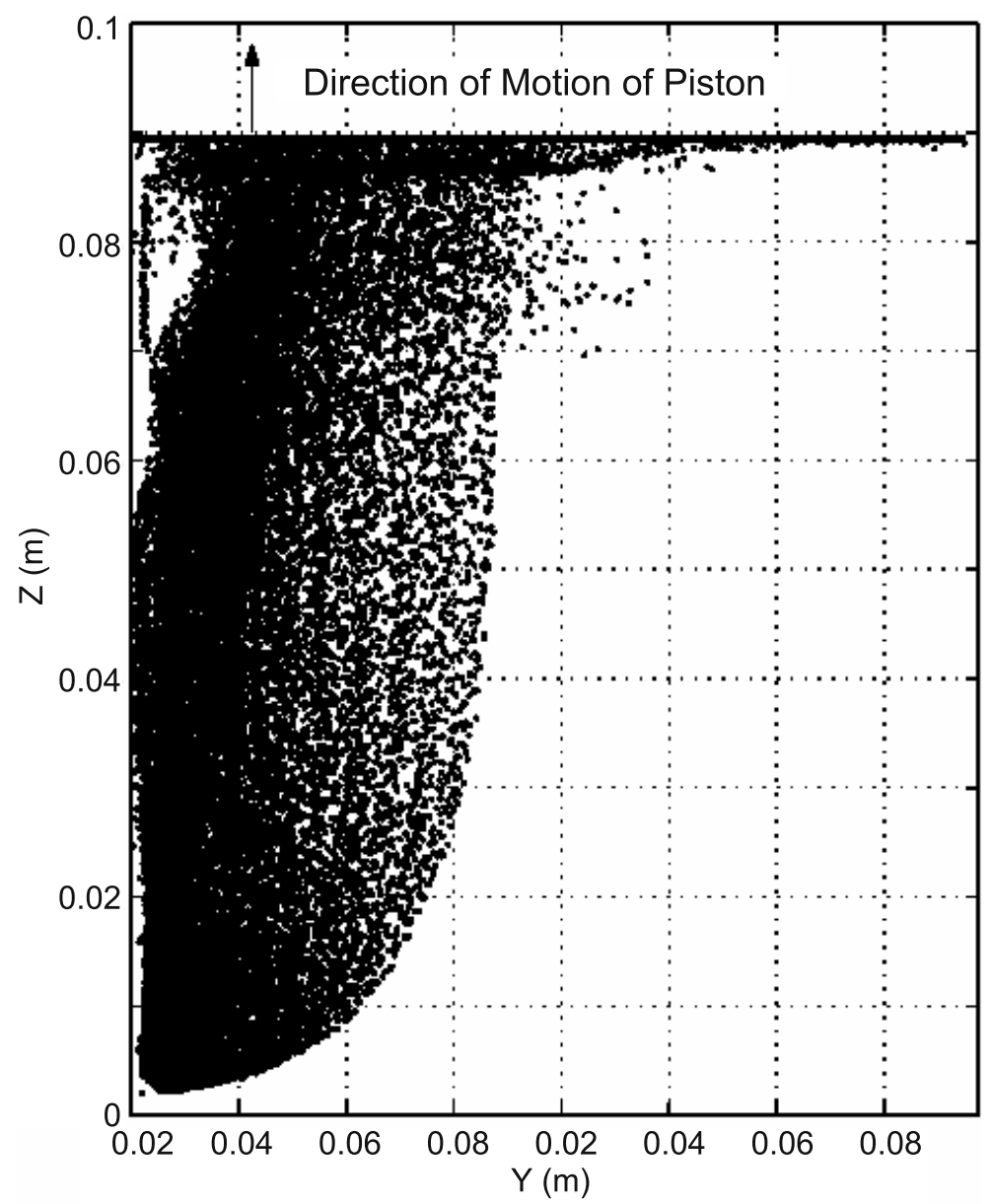

Figure 9. Case 1: Overall view of the spray indicating all droplet positions inside the cylinder at $137 \cdot 8^{\circ}$ ATDC.

in part (b). Thus, experimental results seem to confirm predictions regarding spray structure. The spray shown in part (c) is observed to be a cone-shaped structure.

At $1.2 \mathrm{~ms}$ after SOI, the structure and shape remains same as shown in figures 4(b) and (c) with the smaller droplets dispersing more at the corners of the spray giving a mist like appearance and a more evident toroidal shape in the central region. Again, the SMD plot in part (d) shows that the predicted SMD is low in the central region with the experimental image in part (a) also showing a corresponding low intensity of laser light in this region. Figure 5 shows the comparison of predicted spray tip penetration with the experiment. The experimental spray tip penetration is based on a certain minimum light intensity at the spray tip which normally accounts for $95 \%$ of total fuel mass at a given instant. The corresponding numerically spray tip penetration at any instant of time is determined based on the penetration of the $95 \%$ of the total fuel mass injected till that time. The initial part compares well. Overall, the comparison is favourable, although there is a slight discrepancy towards the later stage. This is because the penetration is greatly dependent on the 


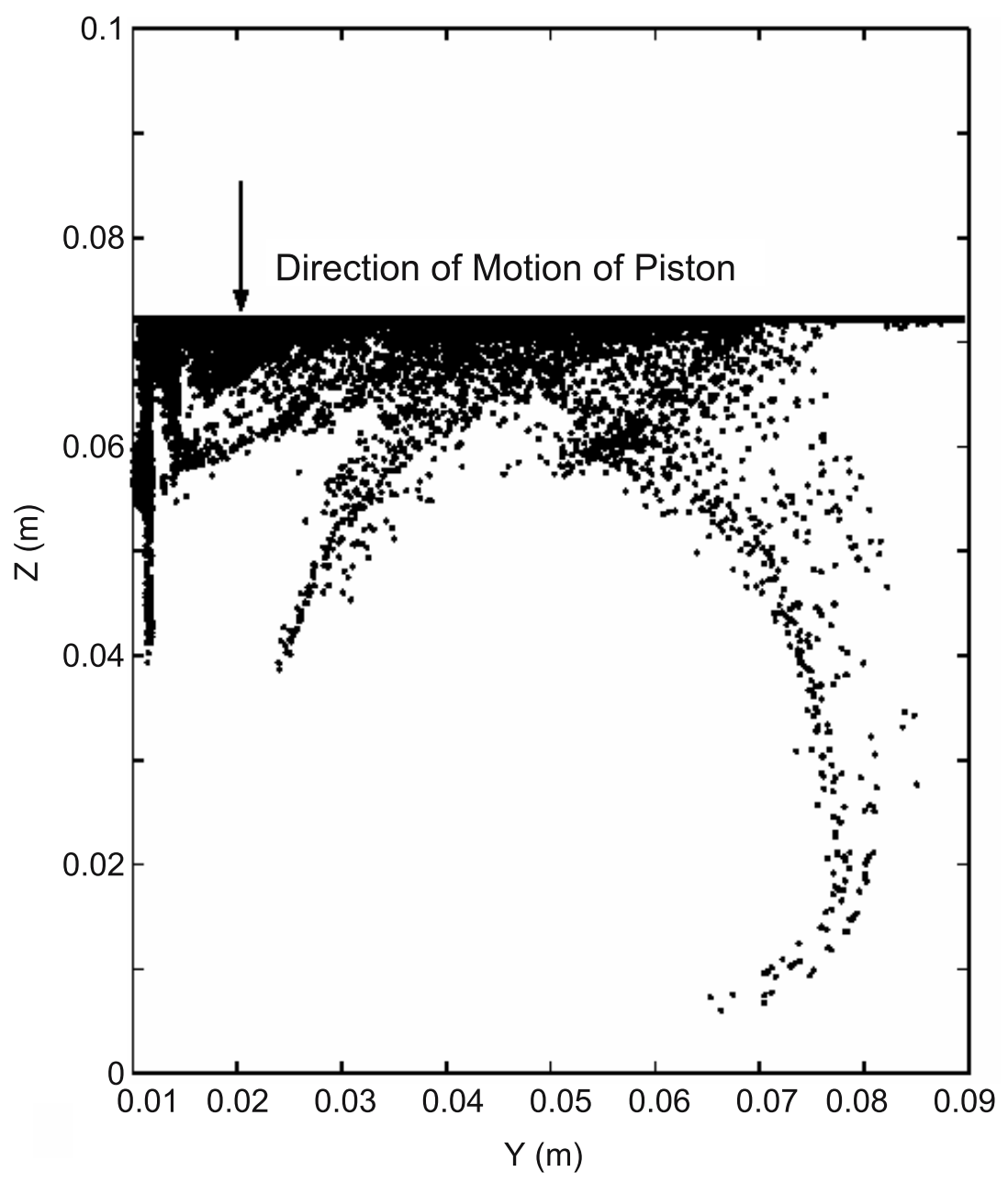

Figure 10. Case 1: Overall view of the spray indicating all droplet positions inside the cylinder at 73. $0^{\circ} \mathrm{ABDC}$.

induced gas motion and the actual droplet size which may slightly differ from the predicted size.

\subsection{Simulation details}

After validation, the aforementioned model was applied to simulate different operating conditions of the engine configuration mentioned earlier. For high and medium loads, the fuel is injected earlier so as to have a near-homogeneous mixture by allowing droplets to mix with air completely and for low loads, fuel is injected late during compression stroke so as to stratify the mixture with less fuel and the near-stoichiometric region becomes the point of ignition. The injection pressure is kept constant and mass of fuel injected is varied depending upon the load. However, the fuel injection rate is maintained constant in all cases. This implies that the structure of the hollow cone spray sheet is similar for all cases. The cases are chosen at 


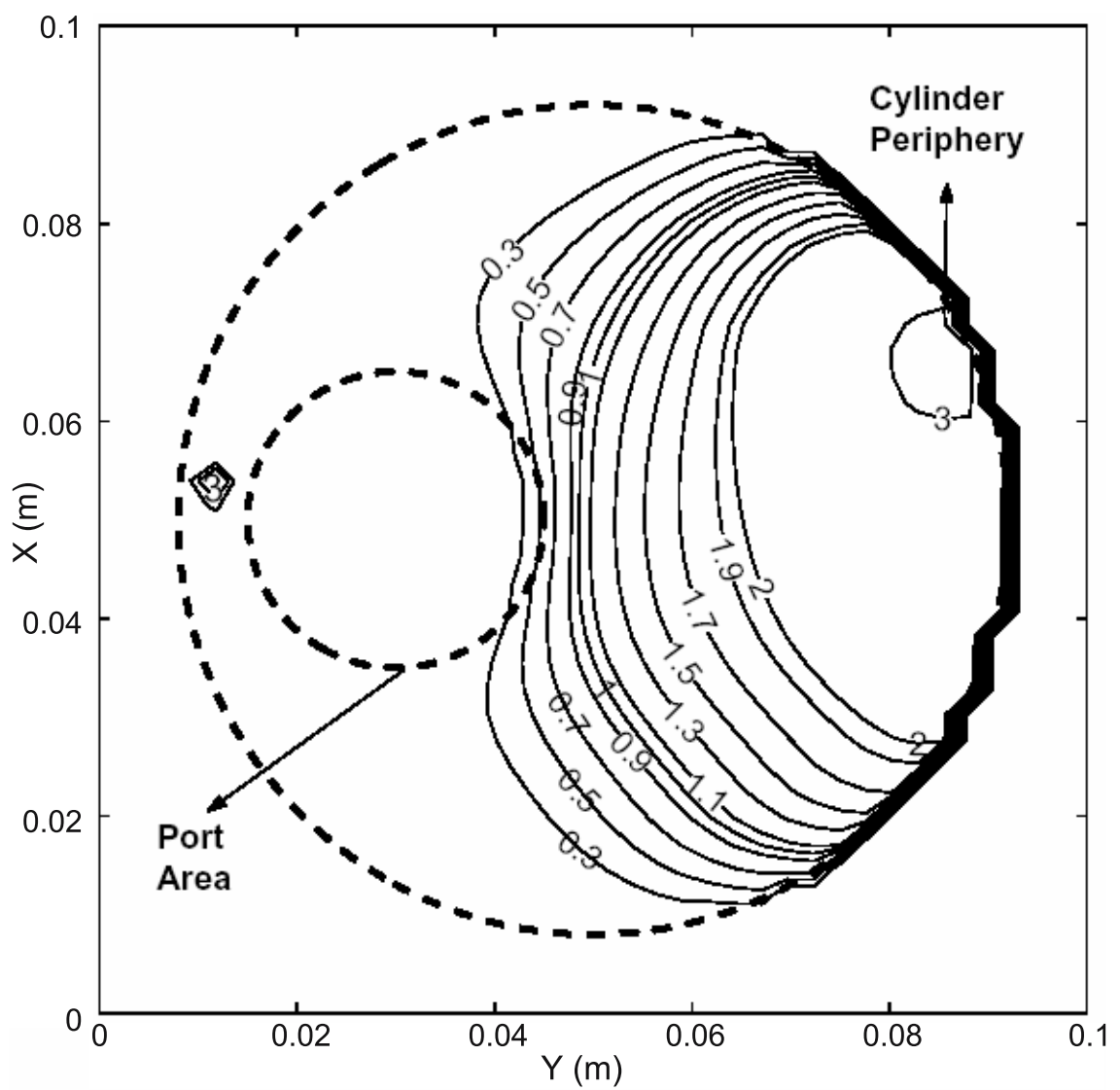

Figure 11. Case 2: Distribution of equivalence ratio in a plane near cylinder head at $18^{\circ} \mathrm{BTDC}$.

different operating conditions based on load and speed in order to cover the engine-operating envelope. In the following sections, the various cases studied are presented with results showing the fuel-vapour concentration distribution inside the cylinder at different crank angles.

The study here is based on different load and speed with engine geometry remaining the same, i.e. flat-piston and flat cylinder head geometry in all cases. The loads speed and the mass of fuel injected are chosen so as to cover the high, mid and low operating points. Table 2 lists the different operating conditions for which the simulations were performed.

Table 2. Different operating conditions of the engine.

\begin{tabular}{lcccc}
\hline Cases & Speed (rpm) & $\begin{array}{c}\text { Fuel injected } \\
(\mathrm{mg})\end{array}$ & $\begin{array}{c}\text { Overall } \\
\text { equivalance ratio }\end{array}$ & Start of injection \\
\hline Case-1 & 4000 & 44 & $1 \cdot 0$ & $90^{\circ}$ ATDC \\
Case-2 & 1000 & 44 & $1 \cdot 0$ & $90^{\circ}$ ATDC \\
Case-3 & 3000 & 33 & $0 \cdot 75$ & $90^{\circ}$ ATDC \\
Case-4 & 3000 & 22 & $0 \cdot 5$ & $90^{\circ}$ BTDC \\
Case-5 & 800 & 18 & $0 \cdot 4$ & $60^{\circ}$ ABDC \\
\hline
\end{tabular}




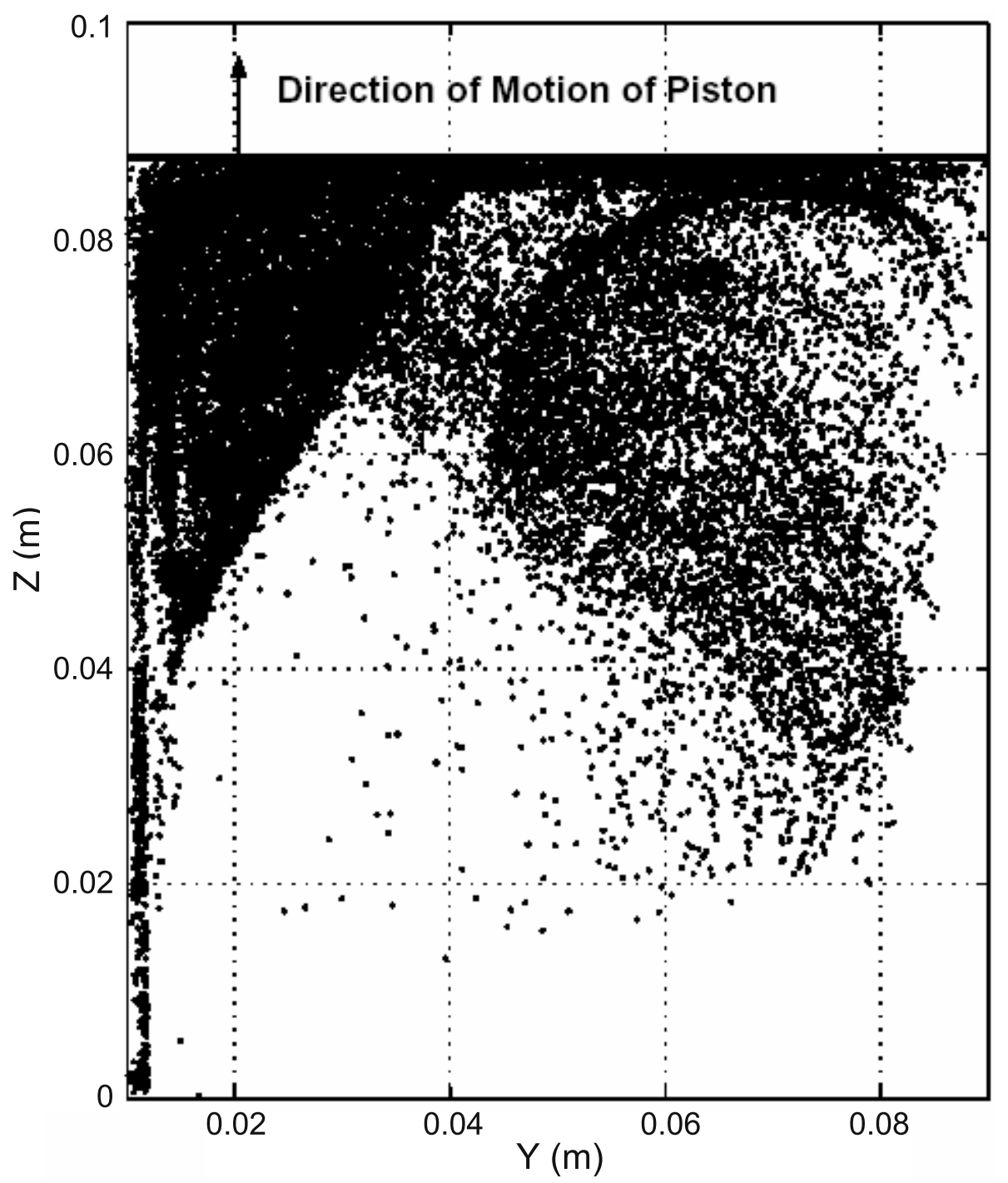

Figure 12. Case 2: Overall view of the spray indicating all droplet positions inside the cylinder at $134^{\circ}$ ATDC.

\section{Results and discussion}

Although the computations give a complete picture of the interaction of the spray with the in-cylinder air-motion as a function of crank angle, results presented here are in terms of the location of near-stoichiometric region in all cases, which can be the possible location of the spark-plug for combustion initiation. In other words, results presented here can serve as a tool in deciding the location of the spark plug. A usual strategy for high load in GDI engines is to inject the fuel early during the intake stroke with overall stoichiometric or slightly rich fuel-air mixture. This allows the droplets to penetrate the entire cylinder and 


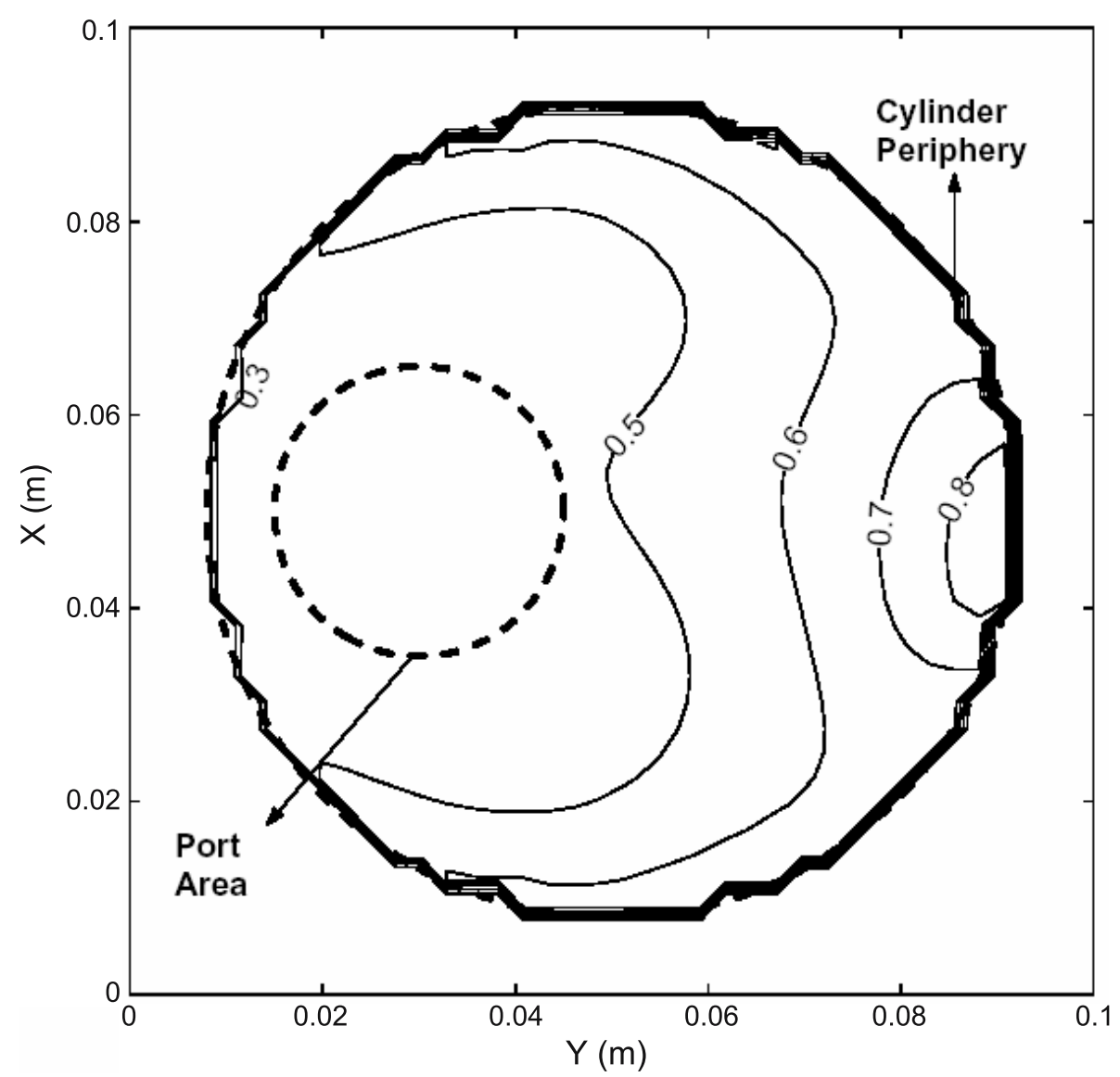

Figure 13. Case 4: Distribution of equivalence ratio in a plane near cylinder head at $18^{\circ}$ BTDC.

fuel vapour to mix better with the air. The desired fuel vapour distribution for high load at the end of compression is to have a homogeneous stoichiometric or slightly rich mixture. This is because for the high load, a homogeneous near-stoichiometric mixture will lead to combustion with less emission and provide the required high mean effective pressure (MEP) for the load requirement. For low loads, the overall mixture is lean as the required MEP is not high. With injecting late, i.e. during the compression stroke, the fuel vapour is stratified, as the fuel vapour does not have sufficient time to mix with the in-cylinder air. In the stratified mode, soot formed due to presence of rich mixture zones in the stratified mixture, will get burned due to the excess air surrounding the vapour-air mixture. Cases-1 and 2 represent the high load case with maximum fuel injected to obtain overall equivalence ratio as stoichiometric. Case- 1 is at high speed of $4000 \mathrm{rpm}$ and Case- 2 is operated at a low speed of $1000 \mathrm{rpm}$.

In Case-1, we study the fuel-air mixing at high load and high speed, i.e., overall equivalence ratio of 1.0 and speed of $4000 \mathrm{rpm}$. In this analysis, $44 \mathrm{mg}$ of fuel was injected at a crank angle of $90^{\circ}$ ATDC. The duration of injection was $2.6 \mathrm{~ms}$ and the injection pressure was assumed to be 60 bars. The droplets are observed to impinge on to the piston surface. This is because the inlet air temperature is low and hence, the droplet vaporization is not substantial. 


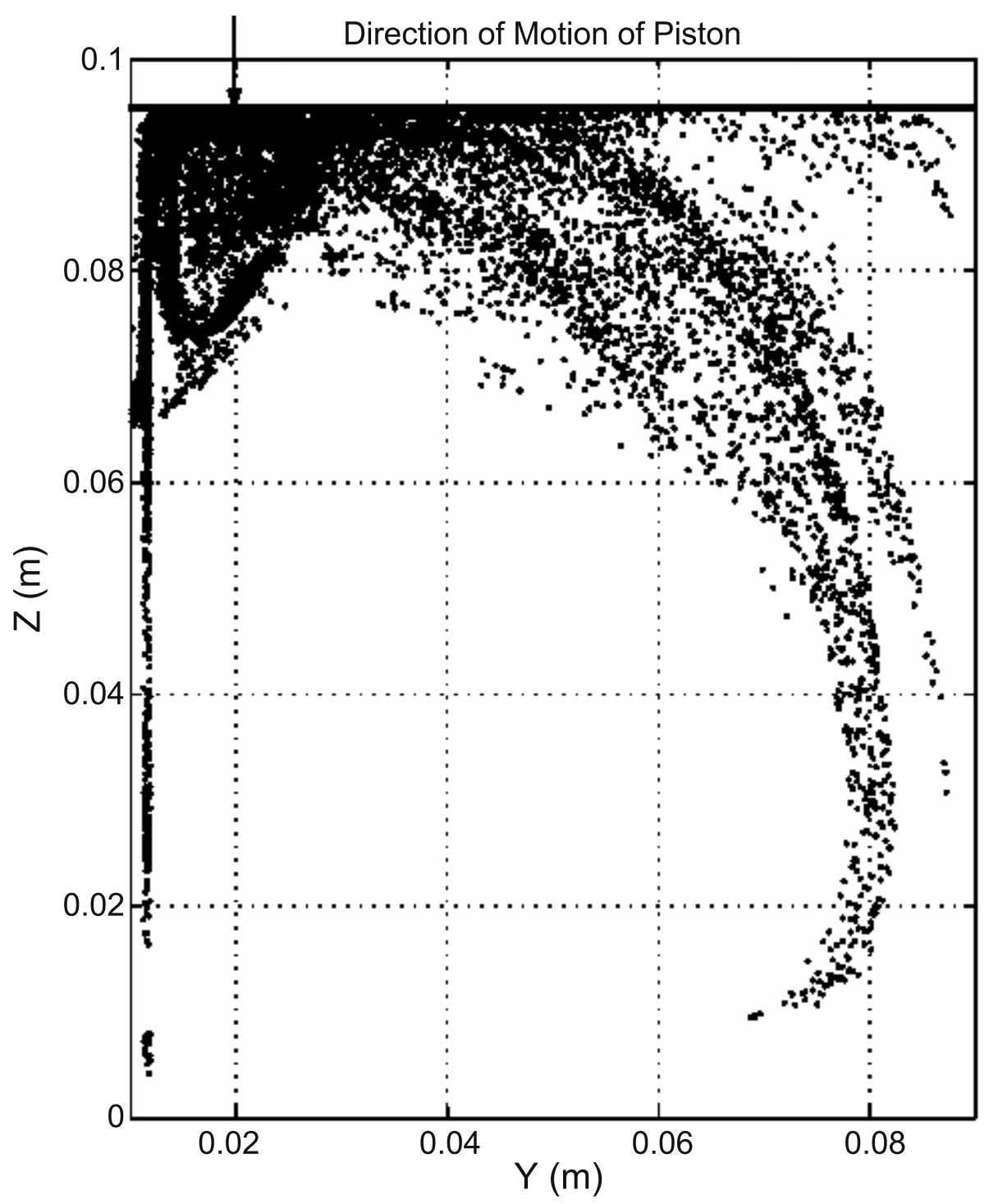

Figure 14. Case 4: Overall view of the spray indicating all droplet positions inside the cylinder at $25 \cdot 2^{\circ} \mathrm{ABDC}$

Figure 6 shows distribution of mixture equivalence ratio near the cylinder head at $18^{\circ}$ BTDC. This distribution is in a plane $1 \mathrm{~mm}$ below the cylinder head surface and will be the plane of investigation for other cases as well in this work. The charge distribution is stratified and the mixture is observed to be stoichiometric to slightly fuel-rich in a narrow stretching from one end of the cylinder to the other. Figure 7 shows the distribution in a plane near the piston surface. The distribution shows rich mixture near the piston surface. This is because, most of the droplets impinge on the piston surface and the resulting break-up and evaporation of droplets causes this region to be fuel-rich. Figure 8 shows the distribution of equivalence ratio in a plane passing through the cylinder axis, injector and the axis of inlet port. This plane 


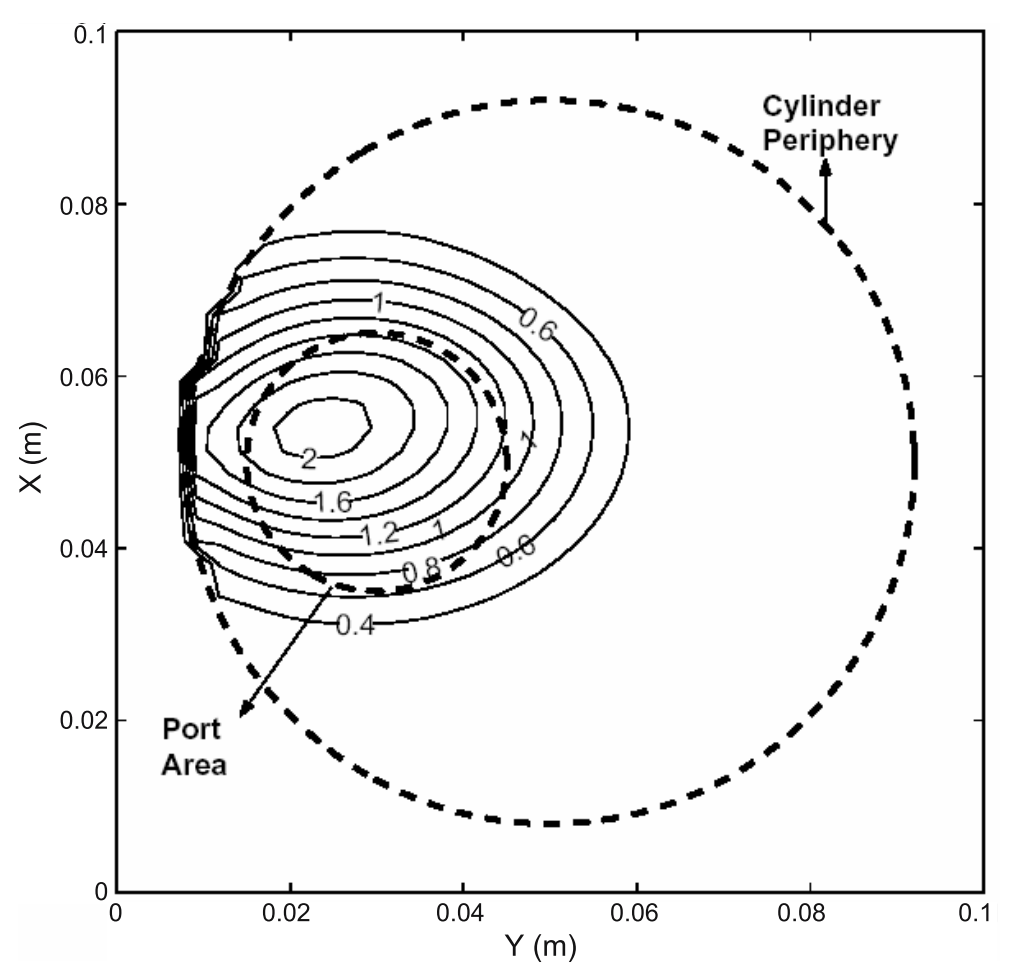

Figure 15. Case 5: Distribution of equivalence ratio in a plane near cylinder head at $18^{\circ} \mathrm{BTDC}$.

will be referred to as the central plane in all subsequent discussions. The equivalence ratio distribution in the central plane shows that the fuel-vapour is stratified and continues to mix with the in-cylinder air. The overall view of spray structure at a crank angle of $137 \cdot 8^{\circ}$ ATDC is shown in figure 9. It is observed that most of the droplets continue to impinge on the piston surface. Some droplets are observed to be trapped in a vortex in the upper-left corner of the cylinder. From figure 10, it is observed that at a later crank angle, the tumble motion of the air drives the droplets, which is intense at this speed. The droplets continue to travel along the cylinder boundary and evaporate simultaneously. The smaller droplets evaporate faster.

Next, we consider the case where the engine experiences high load at low speeds, equivalent to a situation when the vehicle climbs a steep incline. The engine speed has been selected as $1000 \mathrm{rpm}$ and the overall equivalence ratio $(\Phi)$ is assumed to be stoichiometric. The fuel is injected at a crank angle of $90^{\circ}$ ATDC. Again, the pressure of injection is maintained at 60 bars as in the previous case and the fuel is injected for $2.6 \mathrm{~ms}$. Figure 11 shows the predicted fuel vapour distribution near the cylinder head at $18^{\circ} \mathrm{BTDC}$, expressed in terms of equivalence ratio. The mixture is in a stratified form near the cylinder head. The maximum equivalence ratio is observed to be 3 near the right extreme of the cylinder head periphery. The near-stoichiometric mixture region is again formed in a narrow band stretching across the cylinder as shown by the $\Phi=1.0$ contour. The structure of this region is similar to that in Case-1, except that in Case-1, it was moving away from the injector whereas in Case-2, it is moving towards the injector. Thus, the near-stoichiometric region seems to be in the same location in Case- 1 and Case-2. 


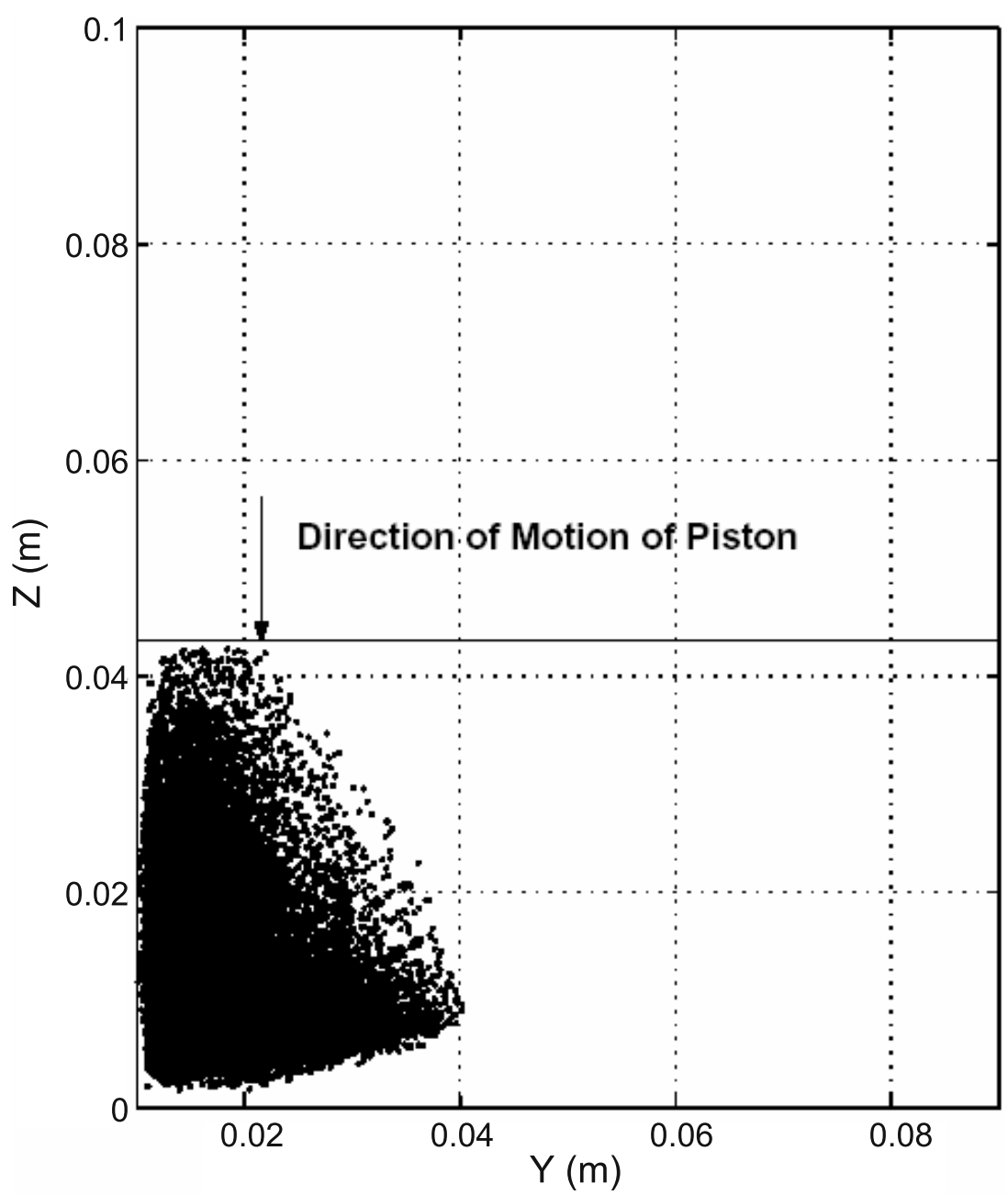

Figure 16. Case 5: Overall view of the spray indicating all droplet positions inside the cylinder at $111 \cdot 6^{\circ}$ ABDC.

Since most of the droplets impinge on to the piston wall as observed in figure 12 at $134^{\circ}$ ATDC, the droplets and vapour continue to travel along the cylinder surface to reach back to the cylinder head. Hence, the mixing is not sufficient enough to obtain a homogeneous near-stoichiometric condition. The deflection of spray from its original direction and the effect of the tumble motion on the spray can be easily visualized in figure 12 . The smaller droplets have already evaporated and the mixing of vapour and air continues, though the maximum concentration remains near the walls signifying poor mixing in this region.

Next, Case-3 is simulated which involves medium load and medium speed and an overall equivalence ratio of $0 \cdot 75$. The engine speed considered here is $3000 \mathrm{rpm}$. The fuel is injected at a crank angle of $90^{\circ}$ ATDC. Other injection details are same as in the previous case. The 
maximum equivalence ratio is around 0.85 near the cylinder head as shown in figure 13 , which also shows the $\Phi$-contours in a plane near the cylinder head. There is no stoichiometric mixture near the cylinder head. In this case, the overall equivalence ratio was around 0.75 , and hence the mixture equivalence ratio is low near the cylinder head. This is also due to early injection of fuel that allows the fuel droplets to mix more and cause a near-homogeneous mixture. This suggests that by reducing the effective timing of mixing of fuel droplets with in-cylinder air, we can create more stratified charge and can obtain at least a certain portion with near-stoichiometric mixture. Hence, in this case, more delay in injection could have resulted in portion of region near cylinder head to be near-stoichiometric. Figure 14 shows that at around $25 \cdot 2^{\circ} \mathrm{ABDC}$, the tumble motion drives the spray structure after impingement and the droplets travel along the cylinder boundary. A corner vortex is again observed as in earlier cases with some droplets getting trapped in it. Since the speed is more than the previous case, the droplets do not have sufficient time to travel all the way back to the cylinder head and many droplets impinge on the piston surface.

The conditions discussed so far represented high and medium loads at different engine speeds. In all the conditions so far, the fuel was injected early into the suction stroke of the engine. However, the engine at low loads and at idling responds differently and hence the fuelinjection strategy needs to be different. In case of low loads, the strategy is to inject the fuel late during compression stroke and get a stratified mixture with a near-stoichiometric ratio near the spark plug. In this particular case (Case-4), engine experiences low load at medium speed and is operated very lean at $\Phi=0 \cdot 5$. The engine speed selected is $3000 \mathrm{rpm}$. The fuel is injected at $68.4^{\circ} \mathrm{ABDC}$, i.e., during the compression stroke.

In this case, the maximum equivalence ratio obtained near the cylinder head is around $2 \cdot 0$, which is near the fuel injector itself. This is observed from figure 15 , which shows the $\Phi$-contours near the cylinder head at a crank angle of $18^{\circ}$ BTDC. The near-stoichiometric mixture is observed to be in a circular region surrounding the inlet port. The maximum equivalence ratio inside the whole cylinder domain is around 3.5 . This signifies that the mixture is extremely stratified. Unlike in previous cases, the near-stoichiometric mixture is located not in the region opposite to the port area of the cylinder head, but in a diametricallyopposite region. This is because the tumble intensity is low compared to previous cases and this affects the droplet motion and vapour mixing. Thus, with the low intensity tumble motion at this stage, the droplets do not penetrate the other side of the cylinder head. This also leads to less distortion of spray structure due to low tumble motion as against the previous conditions and hence, more impingement on the piston surface is observed. Figure 16 represents the overall spray structure at $111.6^{\circ} \mathrm{ABDC}$. The spray is observed to cover a larger volume than in the previous cases of early injection. This is because the spray does not get as deflected as in previous cases owing to the weak tumble motion. It is clear from the figures that the droplets evaporate in a region near the injector location as opposed to the previous cases of early injection where the droplets travel in a loop and evaporate in the region opposite to the injector location. Hence, for the condition of low load and medium speed, the nearstoichiometric mixture is found near the injector itself.

In Case-5, we study fuel-air mixing at the idling condition. The engine speed is assumed to be $800 \mathrm{rpm}$ and the overall equivalence ratio is $0 \cdot 4$. The fuel is injected very late at around $60^{\circ}$ BTDC, i.e., during the later stages of the compression stroke. During idling, the strategy is similar to the previous case, where the fuel is injected late and a stratified distribution with a near-stoichiometric ratio is ensured near the vicinity of spark plug. This ensures running of the engine at idling speed, with a saving in fuel and the overall benefit of low emission as the overall mixture is lean. Figure 17 shows the concentration contours at $18^{\circ} \mathrm{BTDC}$ in a plane 


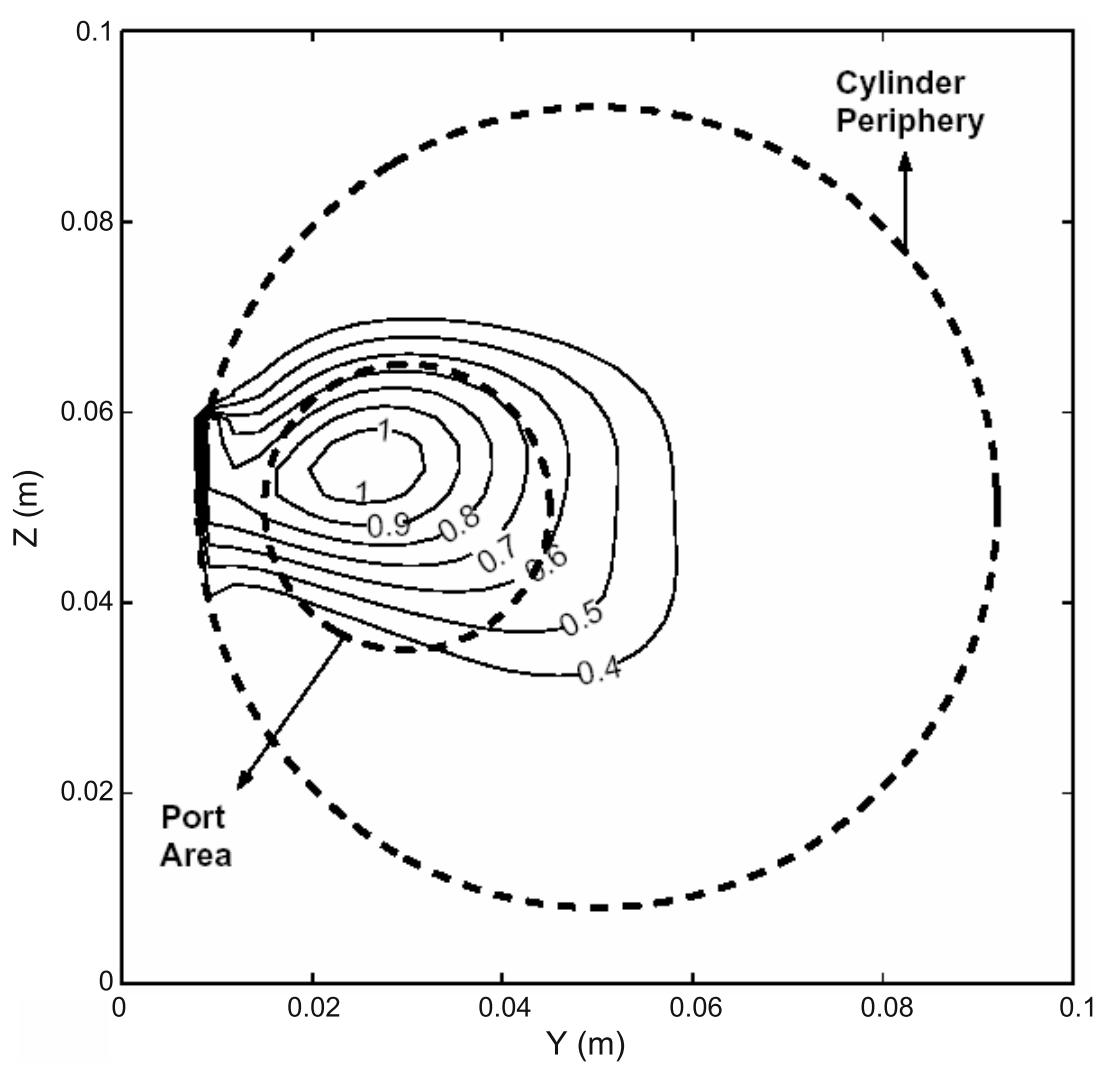

Figure 17. Case 6: Distribution of equivalence ratio in a plane near cylinder head at $18^{\circ}$ BTDC.

near cylinder head. Maximum value of mixture equivalence ratio in figure 17 is 1.05 near the cylinder head in a region around the injector location itself. The maximum equivalence ratio inside the cylinder is 4.05 near the piston surface. This shows that the mixture is very much stratified and is observed to mostly occupy the injector side of the cylinder. This can be observed from figure 18 which shows the overall spray structure at $49 \cdot 8^{\circ}$ BTDC. The tumble motion does not affect the spray, as the intensity of the tumble motion is very low at these crank angles. Hence, the spray more or less remains towards the injector side of the cylinder and continues to evaporate. Thus, the near-stoichiometric mixture is found near the injector itself, at idling condition.

In summary, a range of engine operating conditions for direct fuel injection indicate interesting and very different fuel-air mixing patterns. The observations seem to indicate that for high load conditions, a definite region can be identified for locating the spark plug once the ignition timing is selected. However, for low load conditions, an altogether different region seems to be appropriate. A possible solution to this conflicting requirement for this simple, flat-piston geometry engine could be utilization of dual spark plugs. For high load conditions, the spark plug could be located in a region around the center of the cylinder. To achieve a near-stoichiometric region for the medium load in the same region as that of the high load, the fuel injection could be delayed relatively. For low loads, a second spark plug located slightly offset from the injector could initiate combustion. Thus, sat- 


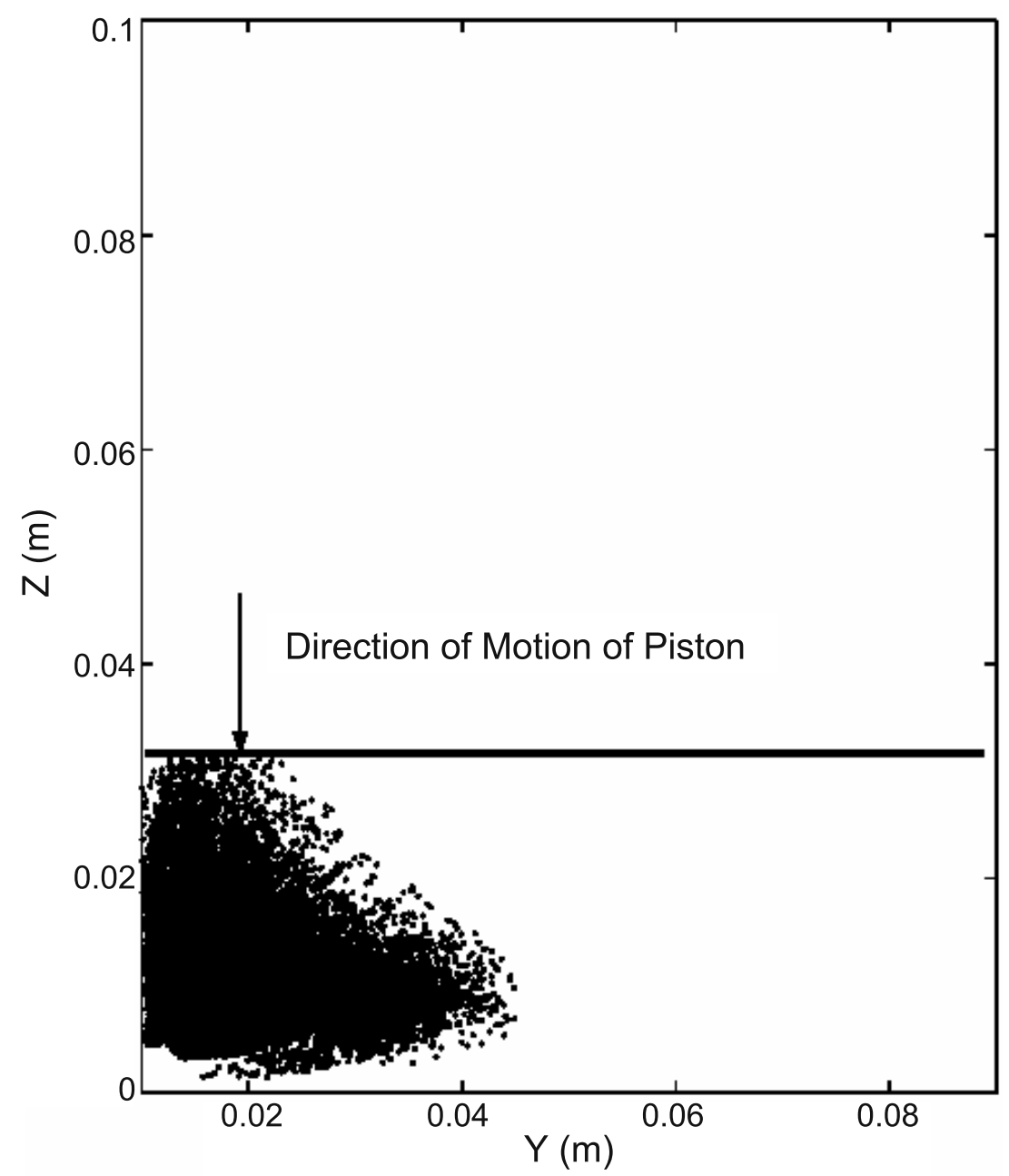

Figure 18. Case 6: Overall view of the spray indicating all droplet positions inside the cylinder at $49 \cdot 8^{\circ} \mathrm{ABDC}$.

isfactory ignition and energy conversion would then be ensured for any engine operating condition.

\section{Conclusions}

A Lagrangian-drop Eulerian-fluid based model has been developed and applied to the incylinder spray-air motion interaction in a conventional spark ignition engine under direct injection conditions. The Taylor Analogy Breakup (TAB) model for modelling the hollow cone spray and appropriate models for droplet impingement, drag and evaporation are used. Moving boundary algorithm and two-way interaction between both phases are implemented. Engine simulations were performed for various cases of speed and load. Fuel droplet trajectories in the pre-combustion phase along with spatial and temporal fuel-air mixing distributions 
were predicted for each case. The simulations indicate interesting and very different fuel-air mixing patterns for different loads. The model developed serves as a useful tool in design and optimization of a direct fuel injection retrofit for a conventional SI engine, with regards to cylinder geometry, injector and spark plug location. The results show that in order to extend the efficiency and low-emission advantages of the direct fuel injection concept to conventional SI engines, a novel ignition strategy such as the dual spark plug configuration could be a suitable option.

\section{References}

Bakshi S, Ravikrishna R V 2003 Multi-dimensional simulation of the air-flow and fuel transport process in the air-assisted injection system of a new, low-emission two-stroke engine, Proc. Instn. Mech. Engrs., vol 217, Part D: J. Automobile Eng. 383-392

Doormal J P V, Raithby G D 1984 Enhancements of a SIMPLE method for predicting incompressible fluid flows. Num. Heat Transfer 7: 147-163

Ferziger J H, Peric M 2003 Computational methods for fluid dynamics, Springer, $3^{\text {rd }}$ edition

Han Z, Fan L, Reitz R D 1997 Multi-dimensional modelling of spray atomization and air-fuel mixing in a direct-injection spark-ignition engine. SAE Paper 970884

Heywood J B 1988 Internal Combustion Engine Fundamentals, McGraw Hill, Inc.

Iwamoto Y, Noma K, Nakayama O, Yamauchi T, Ando H 1997 Development of gasoline direct injection engine. SAE Paper 970541

Jost K 2001 Fuel stratified injection from VW, Automotive Eng. Int., Society for Automotive Engineers (SAE), USA

Naber J D, Reitz R D 1988 Modelling of engine spray/wall impingement. SAE Paper 880107, 1-23

O'Rourke P J, Amsden A A 1987 The TAB method for numerical calculation of spray droplet breakup. SAE Paper 872089

Patankar S V 1980 Numerical heat transfer and fluid flow, (Hemisphere Publications)

Senda J, Kobayashi M, Iwashita S, Fujimoto H 1994 Modelling of diesel spray impingement on a flat wall. SAE Paper 941894

Taylor G I 1963 The Shape and acceleration of a drop in a high speed air stream mechanics of fluids Scientific papers of Geoffrey Ingram Taylor, (ed.) G K Batchelor, (Cambridge University Press), Vol. III

Xu M, Markle L E 1998 CFD-aided development of spray for an outwardly opening direct-injection gasoline injector. SAE Paper 980493 